\title{
THE BRITISH JOURNAL OF VENEREAL DISEASES
}

\section{Case report}

On his admission to hospital there was no swelling over the wrists or lower end of the radius and ulna of either side, but slight tenderness was present at these sites.

The Wassermann and Kahn tests were positive. There was neither penile sore, scar nor urethral discharge to be seen, and he did not give any history of sexual exposure. There were no stigmata of congenital syphilis. The glands in the groin were slightly enlarged.

On x-ray examination it was found that the lower half of both ulnae showed new bone formation and a break in the cortex, with rarefaction of the medulla. Periosteal new bone formation was present in the third metacarpal bone. The findings were suggestive of osteomyelitis due to syphilis. Other bones did not show any abnormality.

The condition was diagnosed as being due to late congenital syphilis. Treatment with 40,000 units of penicillin at 3-hourly intervals was given in 60 injections until a total of $2,400,000$ units was reached. X-ray examination after the penicillin treatment showed that the condition had much improved The Wassermann and Kahn tests gave doubtful results. The patient did not complain of any pain nor was there any tenderness present.

I am grateful to Col. N. J. Gai, Officer Commanding I.M.H. Dunkirk, for permission to publish this case record.

Indian Military Hospital,

N. V. RAO,

Dunkirk

Lt.-Col., I.M.S./I.A.M.C.

\section{REVIEWS OF BOOKS}

Penicillin: Its Practical Application. Under the general editorship of Professor Sir Alexander Fleming, M.B., B.S., F.R.C.P., F.R.C.S., F.R.S. 380 pp., 59 illustrations, Butterworth \& Co. (Publishers) Ltd., London, 1946. Price 30s.

The chance contamination of a laboratory culture plate in the year 1928, with the subsequent growth of a mould, was probably no uncommon event then or in any other year. Fortunately for the seething population of the world, Sir Alexander Fleming noted that the staphylococcal colonies on the culture plate in the vicinity of the mould had undergone dissolution, and he thought that " this was an extraordinary and unexpected appearance and seemed to demand investigation." Sir Alexander's impelling curiosity led to the isolation of this mould in pure culture and its identification as a member of a species of the genus penicillium. He noted also that the culture fluid could be diluted several hundred-fold before it lost its property of inhibiting the growth of the staphylococcus. The story of the discovery of penicillin by Fleming has been told and retold in the medical and lay press of the world; but nowhere has there appeared an account which so deeply arouses our interest and pride in this British achievement as does the engrossing chapter on the history of the discovery and development of penicillin from the pen of Sir Alexander himself.

It has been unusual for the busy doctor to express more than a perfunctory interest in the manufacturing processes which finally provide the medicaments of his daily practice. In the process of the large-scale manufacture of penicillin, however, none can fail to be interested ; and, now, since the end of World War II, when the rigorous wartime secrecy can be largely dispensed with, many a busy and tired medical practitioner can pass a thoroughly enjoyable hour in reading the profusely illustrated chapter on the chemistry and manufacture of penicillin, by A. L. Bacharach and B. A. Hems.

A considerable knowledge of the various methods of using penicillin to kest advantage is an essential basis of our judicious administration. For this purpose we need an understanding of the effects of continuous and intermittent injections and of the local administration of penicillin, of its diffusion and distribution in the body tissues and fluids and of its excretion from the body -all of which are dealt with fully in the informative chapter by L. P. Garrod on the pharmacology of penicillin.

Readers of the Journal will have a particular interest in the section on the use of penicillin in venereal disease therapy, contributed by G. L. M. McElligott. It will be, perhaps, a little disappointing to venereologists to find only 11 pages, in this book of 380 pages, devoted to an account of the use of penicillin in gonorrhoea and syphilis. One cannot help feeling that the space allowed for this purpose is somewhat disproportionate to the present-day importance of these scourges, which, it is hoped, may ultimately be controlled by this important new therapy. Into this short space, however, is condensed a good deal of the rapidly accumulating knowledge of the use and limitations of penicillin treatment in these two venereal diseases. Much of this information is based upon the experience of treatment of cases in the armed Forces. In gonorrhoea, after initial trials of 3-hourly injections, the period of treatment has been reduced from 48 hours to 12 hours and the total dosage stepped up to 150,000-200,000 units-a schedule which results in the cure of about 90 per cent of acute infections. In the treatment of gonorrhoea it is fortunate that, so far, there appears to be no evidence to support the fear of the development of penicillin-resistant strains of the gonococcus. The difficulties of securing in-patient treatment 


\section{REVIEWS OF BOOKS}

for civilians with venereal disease led to the introduction of preparations of penicillin which are characterized by a slow rate of absorption after injection. Of these, calcium penicillin incorporated in beeswax in peanut oil or in ethyl oleate, is advocated for recent gonorrhoea in doses of 100,000 to 150,000 units. Stress is laid upon the danger of masking or delaying concomitantly acquired syphilis when doses of 200,000 units or more are given for gonorrhoea.

The various local and systemic complications of gonorrhoea and their response to penicillin therapy are discussed briefly. The efficacy of penicillin seems to vary. In acute salpingitis and acute prostatitis recovery is rapid and in epididymitis results are somewhat indifferent, whereas acute bartholinitis with abscess formation does not react well until the abscess is drained. Much information of value to the venereologist can be gained from studying the chapters on the use of penicillin in the pyogenic infections of the bones and other tissues, in which the principles of surgical drainage in relation to efficacy of penicillin therapy are thoroughly discussed. Gonococcal iritis is stated to subside rapidly; this effect is unexpected, for, according to A. Sorsby in the chapter on ophthalmology, penicillin, however administered, does not penetrate into the interior of the eye.

Considerable stress is laid on the importance of observation and tests after the apparent cure of gonococcal infection; a period of 3 months is considered to be requisite for men and at least 6 months for women. The appropriate time for the various tests of cure and their details are specified.

The section on the treatment of syphilis describes the rapid healing effect of penicillin upon the primary and secondary lesions of this infection. It is pointed out that the serological response, which does not usually take place until some 4-6 months after treatment, occurs at much the same time as it does after the standard or intensive treatment with the arsenical and bismuth preparations. The earlier total dosage of penicillin, when used alone, of $1,200,000$ units was increased up to $2,400,000$ units in $7 \frac{1}{2}$ days as the routine treatment in the armed Forces of the United States and of Great Britain. Many cases have been thus treated, but it is considered to be premature to foretell the ultimate results, although the immediate effects equal, if they do not excel, those obtained by former methods. The present tendency is to treat early syphilis with 2-3 mega units of penicillin over a period of one week, supported by 10 injections of both an arsenical and a bismuth preparation at weekly intervals. The problem of in-patient treatment for syphilis in civilians is even more formidable than that for cases of gonorrhoea, and therefore a schedule of treatment suitable for out-patients is often required. For this purpose McElligott recommends penicillin (presumably calcium penicillin) suspended in a beeswax-ethyl-oleate medium. Daily doses of 500,000 units are advised for 6 consecutive days, together with arsenical and bismuth therapy prolonged over a period of 40 days.

This first British symposium on penicillin will be found to be of absorbing interest and great value to all medical practitioners who wish to be up to date. The venereologist is well advised to read all the chapters as thoroughly as he does that on venereal disease, for there is much useful knowledge to be gained by studying the reports of the successes and failures of penicillin therapy in other infections.

V.E.L.

Venereal Diseases in General Practice. By Svend Lomholt, M.D. (Copenhagen), O.B.E. 231 pp. ; 127 illustrations, 39 on coloured plates. H. K. Lewis \& Co. Ltd., London, 1946. Price 25s.

This is the first English edition of a textbook which has been generally used in Scandinavian countries, and which reached its third edition in 1945. It is difficult to review a book which was swritten before the recent war but delayed in publication by the German occupation of Denitark. The clinical aspects of the diseases have not greatly changed in the subsequent years, but the advances in methods of treatment render much of the text obsolete. This criticism is overir ne to some extent by the addition of a supplement dealing with modern chemotherapeutic treatnt , it, including the use of penicillin, arsenoxide, sulphathiazole and sulphadiazine. Many will ha $/$ e different views on certain aspects of the treatment advocated; for example, 10 grammes of sulphathiazole daily (p. 224) is not necessary for the cure of gonorrhoea, and 0.06 gramme of arsenoxide is not experimentally equivalent to 0.45 gramme of neoarsphenamine - the " 0 .045 gramme' stated is presumably a misprint (p. 220). In the present changing schemes of treatment, $t$ must be admitted that venereologists are experimenting in an attempt to find an adequate and mproved method of treating these diseases, especially syphilis; the policy of relegating treatment to a supplement can be advocated for more general adoption until the outcome of these experinents is proved. It is unlikely that the knowledge of the clinical side of these diseases will change greatly, and frequent revision of the text could be avoided thereby whilst ensuring clarity of description for the student.

The work suffers from ambiguity as a result of the author's attempt to revise and present the text in English, which to him is a foreign tongue; it would have been better if Professor Lomholt had enlisted the help of an English clinician in clarifying certain statements. This opinion applies particularly to the section on gonorrhoea, partly, perhaps, on account of the author's less close contact with this branch of the subject. The reader gains the impression that many of the opinions are the result of information obtained from published work, for they do not carry the conviction or continuity of the personal views expressed in earlier sections of the book.

An unconventional and attractive cover and a quality of paper seldom found in recent English publications are notable features of this book. The illustrations are numerous and excellent, 